НАУКИ О ЗЕМЛЕ

"НАУКА. ИННОВАЦИИ. ТЕХНОЛОГИИ", №4, 2019

25.00 .29

ФИЗИКА АТМОСФЕРЫ И ГИДРОСФЕРЫ

УДК $551.511 .632+551.511 .639$

Частухин А.B.,

Двоеглазов С.М., Ким Н.С.,

Корнеев В.П.
АНО (Агентство атмосферных технологий),

г. Москва,

Россия

E-mail: attech@mail.ru

\title{
МЕТОДИЧЕСКИЕ ОСОБЕННОСТИ ОЦЕНКИ ЛЬДООБРАЗУЮЩЕЙ ЭФФЕКТИВНОСТИ ПОЛНОРАЗМЕРНЫХ НАЗЕМНЫХ АЭРОЗОЛЬНЫХ ГЕНЕРАТОРОВ
}

Введение:

Материалы и методы

актуальность исследования обусловлена необходимостью совершенствования методик исследования льдообразующей эсрфективности, так как существующие методики по определению эффективности не позволяют получать достаточно полную информацию о льдообразующей эффективности полноразмерных наземных аэрозольных генераторов. Цель статьи заключалась в получении данных о выходе льдообразующих частиц для полноразмерных наземных аэрозольных генераторов при разных скоростях ветра в приземном слое.

исследований: использована лабораторная методика, основанная на применении малого аэродинамического стенда, которая позволяет моделировать условия естественного разбавления аэрозольной струи при работе генератора.

Результаты исследований

и их обсуждение: получены зависимости пьдообразующей эфффективности генераторов от величины начальной скорости разбавления концентрации аэрозольных частиц, а также от степени диафрагмирования поверхности горения пиротехнического состава. Выявлено, что при малой скорости разбавления концентрации аэрозольных частиц (около 1,5 м/c ) степень диасрагмирования поверхности горения пиросостава не оказывает существенного влияния на эффективность генератора.

Выводы: $\quad$ авторами сделан вывод, что при малой скорости разбавления начальной концентрации аэрозольных частиц льдообразующего реагента, свойственной наземным генераторам, броуновская коагуляция льдообразующих частиц приводит к быстрому уменьшению общей концентрации частиц в струе, необходимо учитывать при разработке современных методик. Показано, что дпя пиротехнических наземных генераторов следует применять пиротехнические элементы без диафрагмирования поверхности горения пиросостава, что значительно упрощает конструкцию пироэлемента.

Ключевые слова: скорость разбавления концентрации аэрозольных частиц, льдообразующий реагент, броуновская коагуляция, генератор льдообразующего аэрозоля, аэродинамический стенд, степень диафрагмирования поверхности горения состава. 
Chastukhin A.V., Dvoeglazov S.M., Kim N.S., Korneev V.P. "Agency of atmospheric technologies", Moscow, Russia

E-mail: attech@mail.ru

\section{Methodological Features of the Assessment of the Ice-Forming Efficiency of Full-Sized Ground-Spare Aerosol}

Introduction: the relevance of a research is caused by need of improvement of techniques of a research of ice-form efficiency as the existing methods of determination of efficiency do not allow to obtain rather full information on ice-form efficiency of full-size land aerosol generators. The purpose of article consisted in data acquisition about an exit of ice-form particles for full-size land aerosol Materials and methods generators at different speeds of wind in a ground layer.

of research: the laboratory technique based on use of the small aerodynamic stand which allows to model conditions of natural dilution of an aerosol stream

Results of research during the operation of the generator is used.

and their discussion: dependences of ice-form efficiency of generators on the size of initial speed of dilution of concentration of aerosol particles and also on extent of masking of a surface of burning of pyrotechnic structure are received. It is revealed that at a small speed of dilution of concentration of aerosol particles (about $1.5 \mathrm{~m} / \mathrm{s}$ ) extent of masking of a surface of burning of pyrostructure has no significant effect on efficiency of the generator.

Conclusions: $\quad$ authors drew a conclusion that at a small speed of dilution of the initial concentration of aerosol particles of ice-form reagent peculiar to land generators, Brownian coagulation of ice-form particles leads to fast reduction of the general concentration of particles in a stream, it is necessary to consider when developing modern techniques. It is shown that it is necessary to apply pyrotechnic elements without masking of a surface of burning of pyrostructure to pyrotechnic land generators that considerably simplifies a pyroelement design.

Key words: $\quad$ speed of dilution of concentration of aerosol particles, ice-form reagent, brownian coagulation, generator of an ice-form aerosol, aerodynamic stand, extents of masking of a surface of burning of structure.

\section{Введение}

Большое влияние на количество аэрозольных частищ льдообразующего реагента образующихся в атмосфере в результате работы генератора оказывают его конструктивные параметры и условия истечения парогазовой смеси. Так за счет изменения конструкции генератора можно существенно влиять на такие внутри камерные параметры как давление, температура, время нахождения аэрозольных частиц под воздействием повышенного давления и температуры, которые в свою очередь увеличивают или уменьшают льдообразующую эффективность генератора, а зная влияние условий (скорость обдува, температура окружающей среды и т.п.) при которых происходит истечения парогазовой смеси в атмосферу наиболее продуктивно применять генератор. В этой связи, для определения влияния внешних условий на 


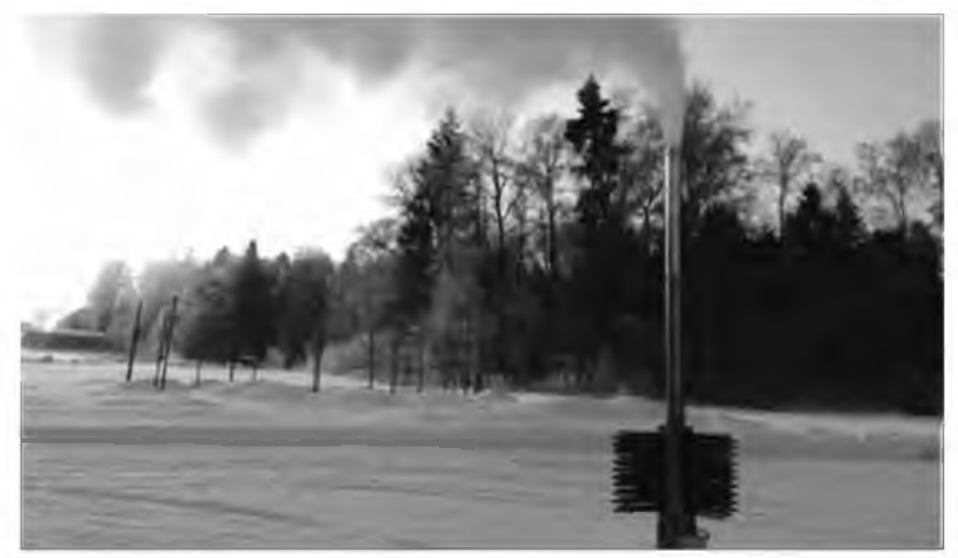

Pnc. 1.

Аэрозольная струя при работе пиротехнического наземного аэрозольного генератора НАГ-07М.

Fig. 1. Aerosol stream during the work of pyrotechnic land aerosol NAG-07M generator.

работу генератора в частности скорости разбавления концентрации аэрозольных частиц льдообразующего реагента при истечении из генератора льдообразующего аэрозоля (далее по тексту - генераторов) парогазовой смеси (скорости обдува) был проведен ряд экспериментов.

Сушественно отличная скорость разбавления конщентрации аэрозольных частиц льдообразующего реагента имеется у самолетных генераторов (скорость обдува 100-570 км/ч) размещаемьх на борту самолетов типа АН12, MB-500 и наземных генераторов (скорость обдува 0-20 км/ч) размещаемых на наземных, стационарных установках.

В существующих стендовых и лабораторных методиках $[1,3,4]$ определения величины выхода активных частиц при испытаниях наземных генераторов влияние скорости разбавления аэрозольной струи не учитывается в полной мере.

Приведено фото аэрозольной струи из пиротехнического наземного аэрозольного генератора НАГ-07М (рис. 1).

Как видно при работе наземных аэрозольных генераторов (рис. 1) аэрозольная струя сохраняется в высококонцентрированном состоянии на достаточном большом расстоянии от генератора из-за относительно небольших скоростей разбавления. В такой струе происходят быстрые процессы взаимной броуновской коагуляции аэрозольных частиц, что приводит к уменьшению общего выхода льдообразующих частиц.

Для проведения испытаний полноразмерных наземных аэрозольных генераторов в АНО «Агентство АТТЕХ» был создан малый аэродинамический стенд, позволяющий моделировать условия естественного разбавления аэрозольной струи при работе генератора. В данной работе приводятся опи- 

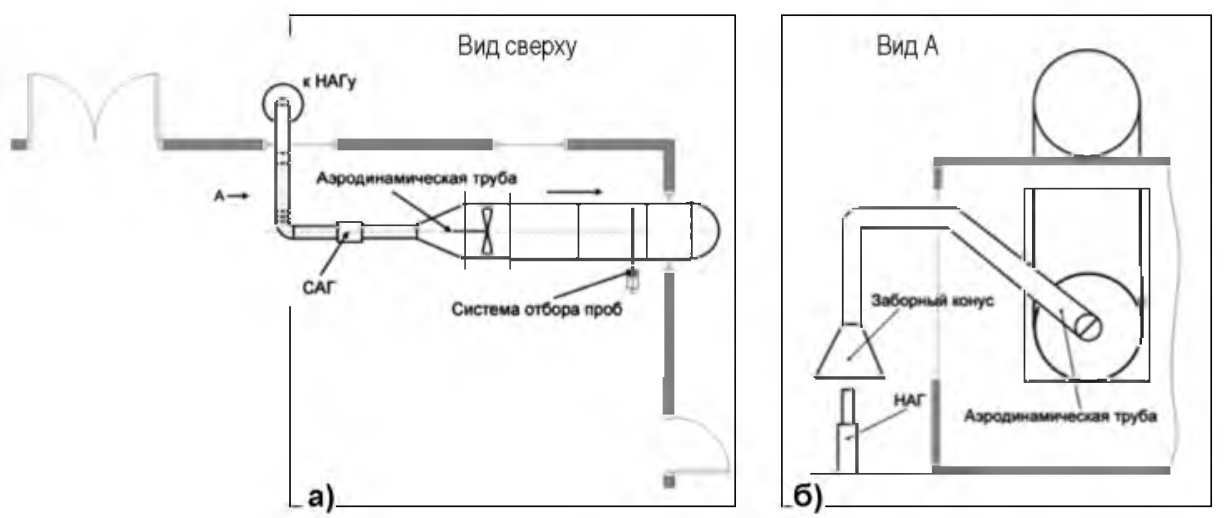

Рис. 2.

Схема аэродинамического стенда:

a) испытания самолетного генератора (при обдуве),

б) испытания наземного генератора (без обдува).

Fig. 2. Scheme of the Aerodynamic Stand: a) tests of the plane generator (when blowing), b) tests of the land generator (without blowing).

сание стенда и результаты исследования на нем льдообразующей эффективности полноразмерных генераторов.

Схема аэродинамического стенда (рис. 2) позволяющая проводить испытания, как самолетных генераторов льдообразующих аэрозолей, так и наземных генераторов. В первом случае самолетный генератор располагается в рабочей части трубы для испытаний при скоростном обдуве (рис. 2a). Во втором случае полноразмерный наземный генератор располагается под входным заборным конусом (рис. 2б). Меняя объемный расход воздуха через сечение аэродинамической трубы, можно моделировать разные скорости разбавления аэрозольной струи из выходной трубы наземного аэрозольного генератора внешним набегаюшим потоком воздуха, т.е. определять выход льдообразующих частиц при разных скоростях ветра в приземном слое.

\section{Материалы и методы исследований}

Методика испытания заключается в следующем. В аэродинамической трубе с помощью вентилятора устанавливается заданные скоростные или расходные характеристики. После включения самолетного или наземного генератора формирующийся льдообразующий аэрозоль поступает в тракт трубы. Затем с помощью пробоотборного устройства через заборную трубку, установленную стационарно в тракте аэродинамической трубы, отбирается дозированная проба аэрозоля, которая вводится в переохлажденный туман, сформированный в облачной камере. Дальнейшая процедура получения данных о льдообразующей эффективности аэрозольного генератора осуществляется по стандартной методике ФГБУ «ЦАО» [4]. 
Ниже приводятся результаты испытаний на льдообразующую эффективность наземных ацетонового и пиротехнического аэрозольных генераторов. Испытания наземных генераторов проводились при моделировании их функционирования при скорости ветра 1,5 м/с.

\section{Результаты исследований и их обсуждение}

На графике (рис. 3) приведены температурные зависимости выхода льдообразующих частиц формирующихся при работе жидкостного наземного генератора с различными ацетоновыми растворами йодистого серебра (AgI).

Полученные данные о льдообразующей эффективности (рис. 3) показали, что в области температур минус $10{ }^{\circ} \mathrm{C}$ и выше они значительно ниже данных, приведенных в других источниках для аналогичных растворов и генераторов $[6,7]$. Такие расхождения, прежде всего, связаны с тем, что в методиках испытаний этих работ корректно не учитывался фактор влияния на конечную величину выхода взаимной броуновской коагуляции на начальном участке распространения аэрозольной струи от генератора.

Известно, что эффективность пиротехнических составов в микромодельных генераторах и самолетных генераторах может сильно зависеть от степени диафрагмирования поверхности горения состава. Однако, как показали проведенные теоретические оценки [2], для случая наземных генераторов влияние этого параметра может быть несущественным на фоне более сильного влияния процесса броуновской коагуляции. Для оценки влияния диафрагмирования на работу наземных генераторов были проведены испытания пироэлементов, как с диафрагмированием поверхности горения, так и с открытой поверхностью горения. В этих экспериментах наземный аэрозольный генератор НАГ-07М устанавливался под заборным конусом аэродинамического стенда. Скорость воздушного потока в рабочей части аэродинамической трубы устанавливалась около $20 \mathrm{~m} / \mathrm{c}$, тем самым моделировалось функционирование НАГ-07М в натурных условиях при скорости приземного ветра 1,5 м/с. Результаты этой серии экспериментов приведены на графике (рис. 4).

Как видно из сравнения кривых на рисунке, величина выхода во всем исследованном температурном диапазоне переохлажденного модельного тумана в обоих случаях находится на одном уровне.

Кроме того, при использовании диафрагмирования поверхности горения было отмечено влияние длины пироэлемента генератора на величину выхода льдообразующих частиц. Так в работе [5] было показано, что при диафрагмировании поверхности горения наблюдается изменение величины выхода по мере продвижения фронта горения пиротехнического состава к задней части генератора. Такая зависимость, прежде всего, связана с тем, что в случае диафрагмирования, во-первых, по мере выгорания состава внутри камеры сгорания генератора меняется температурный режим возгонки йоди- 


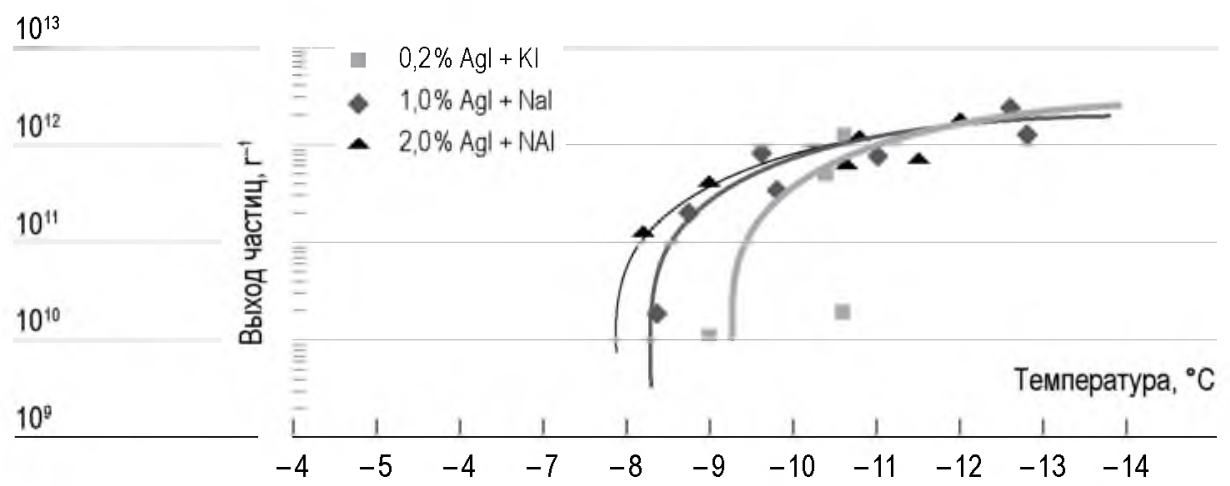

Рис. 3.

Льдообразующая эффективность жидкостного наземного генератора с различными ацетоновыми растворами Agl.

Fig. 3. Ice-form efficiency of the liquid land generator with various acetone Agl solutions.

да серебра и происходит дополнительная диссоциация $\mathrm{AgI}$ на ионы $\mathrm{Ag}^{+}$и I- c последующим их осаждением на стенки камеры и химическим взаимодействием с другими продуктами горения пиротехнической смеси и, во-вторых, с возможным падением концентрации образующихся аэрозольных частиц за счет броуновской коагуляции внутри камеры сгорания.

\section{Выводы}

Проведенные исследования показали, что при работе наземных генераторов, когда разбавление начальной концентрации в аэрозольной струе происходит относительно медленно, основную роль начинает играть броуновская коагуляция льдообразующих частиц, которая приводит к быстрому уменьшению общей концентрации частиц в струе, что необходимо учитывать при проведении оценок эффективности полноразмерных наземных аэрозольных генераторов.

Показано, что для пиротехнических наземных генераторов следует применять пиротехнические элементы без диафрагмирования поверхности горения пиросостава, что значительно упрощает конструкцию пироэлемента.

\section{Библиографический список}

1. Ким Н.С. Исследование влияния условий генерации аэрозоля Agl на его льдообразующую активность // Труды ЦАО. 1980. Вып. 142. С. 89-98.

2. Ким Н.С., Корнеев В.П., Петрунин А.М., Частухин А.В Методы оценки эффективности современных льдообразующих средств воздействия // Сборник статей памяти Н.О. Плауде. 2015. С. 168-184.

3. Ким Н.С., Петрунин А.М., Корнеев В.П., Поносов В.С., Резников М.С. Разработка малогабаритного стенда для определения эффективности пиротехнических средств воздействия // Современные проблемы пиротехники: 


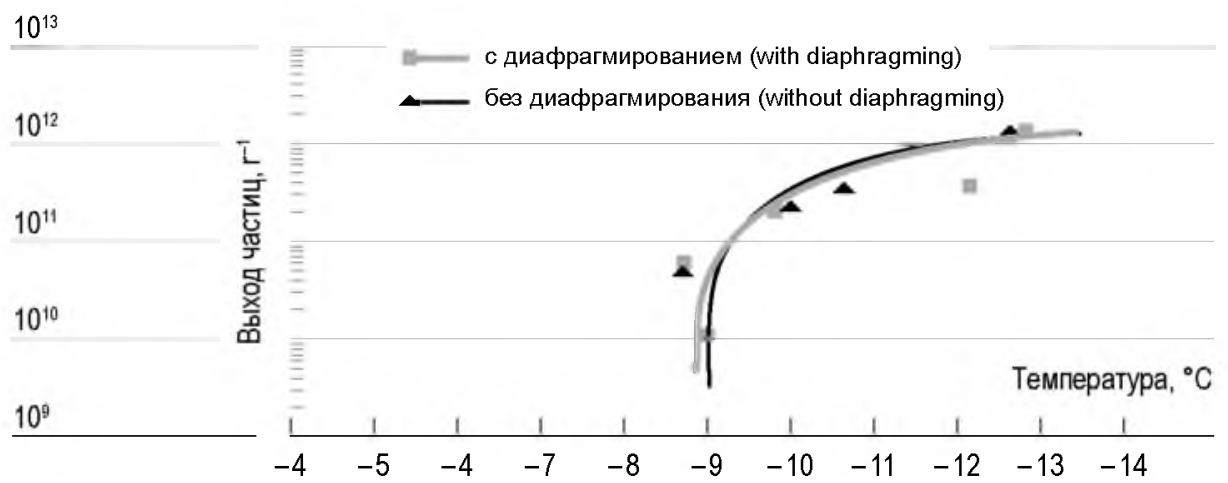

Pис. 4.

Льдообразующая эффективность пиротехнического наземного аэрозольного генератора.

Fig. 4. Ice-form efficiency of the pyrotechnic land aerosol generator.

материалы V Всероссийской научно-технической конференции. Сергиев Посад, 2010. С. 189-196.

4. Методические указания: Методика оценки эфффективности льдообразующих реагентов и пиротехнических составов в лабораторных условиях. РД. 52.11.639-2002. С. 25.

5. Частухин А.В., Ким Н.С., Корнеев В.П., Петрунин А.М., Баззаев Т.В., Данелян Б.Г., Сосникова Е.В. Исследование льдообразующей эффективности полноразмерных жидкостных и пиротехнических наземных аэрозольных генераторов на аэродинамическом стенде ФГБУ «ЦАО» // Доклады Всероссийской конференции по физике облаков и активным воздействиям на гидрометеорологические процессы. Ч. 1. Нальчик, 2017. С. 181-187.

6. Association Nationaled'Etudeet de Luttecontre les Fleaux Atmospheriques «Anelfa»: http://www.anelfa.asso.fr/Article-en-attente.html.

7.Dessens J. Ground experiments in France to prevent hail // J. WeatherModif. 1979. Vol. 11. № 1. P. 4-17.

\section{References}

1. Kim N.S. The study of the influence of conditions of generation of an aerosol of $\mathrm{Agl}$ on its ice-form activity // Works of the Central Administrative District. 1980. Release 142. Page 89-98.

2. Kim N.S., Korneev V.P., Petrunin A.M., Chastukhin A.V Methods of assessment of efficiency of modern ice-form levers // Collection of articles of memory of N.O. Plaude. 2015. Page 168-184.

3. Kim N.S., Petrunin A.M., Korneev V.P., Ponosov V.S., Reznikov M.S. Development of the small-sized stand for determination of efficiency of pyrotechnic levers // Modern problems of pyrotechnics: materials $V$ of the All-Russian scientific and technical conference. Sergiyev Posad. 2010. P. 189-196.

4. Methodical instructions: Technique of assessment of efficiency of ice-form reagents and pyrotechnic structures in vitro. RD. 52.11.639-2002. P. 25.

5. Chastukhin A.V., Kim N.S., Korneev V.P., Petrunin A.M., Bazzayev T.V., Dane- 
Iyan B.G., Sosnikova E.V. The study of ice-form efficiency of full-size liquid and pyrotechnic land aerosol generators at the aerodynamic stand of Federal State Budgetary Institution Central Administrative District // Reports of the All-Russian conference on physics of clouds and active impacts on hydrometeorological processes. Part 1. Nalchik. 2017. P. 181-187.

6. Association Nationaled'Etudeet de Luttecontre les Fleaux Atmospheriques «Anelfa»: http://www.anelfa. asso.fr /A rticle-en-attente.html.

7. Dessens J. Ground experiments in France to prevent hail // J. Weather Modif. 1979. Vol. 11. № 1. P. 4-17.

Поступило в редакцию 23.10.2019, принята к публикации 02.09.2019

\footnotetext{
Частухин Андрей Викторович, главный специалист, АНО «Агентство атмосфрерных технологий», 123242, г. Москва, ул. Дружинниковская, д. 15; тел/фракс: 8 (499) 252-02-41; E-mail: attech@mail.ru; моб. телефон 8(916)257-69-61.

Двоеглазов Сергей Михайлович, ведущий инженер конструктор, АНО «Агентство атмоссрерных технологий», 123242, г. Москва, ул. Дружинниковская, д. 15; тел./факс: 8 (499) 252-02-41; E-mail: attech@mail.ru; моб. телефон: 8(926) 525-76-59.

Ким Николай Сергеевич, доктор физико-математических наук, главный специалист, АНО «Агентство атмосфрерных технологий», 123242, г Москва, ул. Дружинниковская, д. 15; тел./факс: 8 (499) 252-02-41; E-mail: attech@mail.ru; моб. телефон: 8(903) 026-59-98.

Корнеев Виктор Петрович, кандидат технических наук, директор, АНО «Агентство атмосферных технологий», 123242, г. Москва, ул. Дружинниковская, д. 15; тел./ракс: 8 (499) 252-02-41; E-mail: attech@mail.ru; моб. телефон: 8 (985) 761-94-52.
}

\section{About the authors}

Chastukhin Andrey Viktorovich, chief specialist, ANO «Agency of Atmospheric Technologies», 123242, Moscow, Druzhinnikovskaya St., 15; tel./fax: 8 (499) 252-02-41; E-mail: attech@mail.ru; mob. phone: 8 (916) 257-69-61.

Dvoyeglazov Sergey Mikhaylovich, leading design engineer, ANO «Agency of Atmospheric Technologies»; 123242, Moscow, Druzhinnikovskaya St., 15; tel./fax: 8 (499) 252-02-41; E-mail: attech@mail.ru; mob. phone: 8(926) 525-76-59.

Kim Nikolay Sergeyevich, doctor of physical and mathematical sciences, chief specialist, ANO «Agency of Atmospheric Technologies», 123242, Moscow, Druzhinnikovskaya St., 15; tel./fax: 8 (499) 25202-41; E-mail: attech@mail.ru; mob. phone: 8(903) 026-59-98.

Korneev Victor Petrovich, candidate of Technical Sciences, director, ANO «Agency of Atmospheric Technologies»; 123242, Moscow, Druzhinnikovskaya St., 15, tel./fax: 8 (499) 252-02-41;

E-mail: attech@mail.ru; mob. phone: 8(985)761-94-52. 